\title{
ANÁLISE COMPARATIVA DE REDES HIDROLÓGICAS GERADAS A PARTIR DE SUPERFÍCIES HIDROLOGICAMENTE CONSISTENTES
}

Comparative Analysis of Hydrologic Networks generated from Hydrologically Correct Surfaces

\author{
HUGO JOSÉ RIBEIRO \\ NILSON CLEMENTINO FERREIRA
}

Universidade Federal de Goiás

Programa de Pós-Graduação em Engenharia do Meio Ambiente - PPGEMA

Praça Universitária s/n - Setor Universitário - Goiânia - GO - Brasil nclferreira@gmail.com; hgribeirogeo@gmail.com

\begin{abstract}
RESUMO
Um dos principais desafios da análise hidrológica está na caracterização morfométrica e delineamento da rede hidrográfica associada a uma bacia. Neste contexto, objetivou-se a comparação qualitativa e quantitativa de parâmetros geomorfométricos extraídos de redes hidrológicas geradas a partir de superfícies originais, e hidrologicamente consistentes. A priori, determinaram-se dois grupos de informações. O original, derivado das superfícies sem nenhuma correção e o corrigido, derivado das superfícies hidrologicamente consistentes. As informações de drenagem foram extraídas a partir de cada modelo digital de terreno (MDT) e cada modelo digital de elevação (MDE), antes e após a correção, para uma posterior comparação entre as redes estimadas e de referência. As comparações foram feitas na escala de 1:100.000, tomando por base o MDE SRTM de menor resolução espacial. Percebeu-se que as distâncias das redes são inversamente proporcionais a resolução espacial de cada superfície, exceto para o GDEM, que, mesmo com resolução espacial de 30metros, não gerou melhores resultados do que o SRTM com resolução espacial de 90metros. Das superfícies corrigidas a Topodata foi menos sensível a mudança, com uma amplitude da distância média (antes e após a correção) de apenas 24,5 metros. Enquanto o SRTM teve a maior amplitude com 76
\end{abstract}


metros. Os MDTs de alta resolução espacial apresentaram resultados similares, com o maior ajuste para o MDT obtido por levantamento aerofotogramétrico.

Palavras-chave: MDEHC; MDT; Condicionamento de MDE; Comparação.

\section{ABSTRACT}

One of the biggest challenges concerning hydrological analyses is the morphometric characterization and delineation of the hydrographic network associated with a basin. Therefore, this study aimed at comparing the qualitative and quantitative of geomorphometic parameters extracted from hydrological networks produced from original and hydrologically consistent surfaces. At first, were elaborated two datasets were elaborated. The first, denominated original, organized from surfaces without any correction and the second, denominated corrected, derived from the hydrologically correct surfaces. Drainage maps were automatically extracted from each digital terrain model (DTM) and digital elevation model (DEM), before and after corrections for a posterior comparison between the estimated and the reference networks. Comparisons were made considering the 1:100.000scale. It was noticed that the distances between the networks were directly proportional to the spatial resolution of the surfaces data, except for GDEM, which even with spatial resolution of 30meters, presented inferior results than DEM SRTM with spatial resolution of 90meters. The corrected Topodata surface was less sensitive to changes, with an average distance (before and after corrections) of only 24.5 meters. The drainage, extracted from SRTM presented the worst unconformity,76 meters. Finally, the drainage extract from high-resolution DTMs presented similar results, with the beet adjustment for DTM produced from aerial photogrammetry.

Keywords: DEMHC; DTM; DEM Reconditioning; Comparison.

\section{INTRODUÇÃO}

A água é a substância mais abundante da Terra, a principal constituinte de toda vida terrestre. A existência e progresso de uma civilização são diretamente influenciados pela disponibilidade de água durante ociclo hidrológico. A hidrologia é a ciência que trata de todas as fases do movimento da água na Terra, que é de grande importância para a população e o meio ambiente (TE CHOW et al., 1988).

Um dos principais desafios da análise hidrológica está na caracterização morfométrica e delineamento da rede hidrográfica de uma mesma bacia hidrográfica (PIRES et al. 2005). Para Villela e Mattos (1975), o conhecimento de tais elementos é de grande relevância, pois uma vez estabelecidas as relações e comparações entre os índices e dados hidrológicos conhecidos, é possível determinar de forma indireta, os valores hidrológicos em locais desprovidos destas informações.

Atualmente, com o avanço tecnológico no ramo computacional e espacial, é possível extrair informações hidrológicas de forma rápida, reduzindo custos e a subjetividade na extração destas informações. Uma das ferramentas mais eficazes 
para o processamento e gerenciamento destes dados são os Sistemas de Informações Geográficas (SIGs).

O elemento principal, e mais utilizado nos SIGs para esta tarefa é o modelo digital de elevação (MDE), que representa matematicamente as feições do terreno. Existem diversas fontes de MDEs disponíveis na Internet, entre elas o SRTM (Shuttle Radar Topography Mission), GDEM (Global Digital Elevation Model) e o Topodata. No entanto, estes dados não são adequados para uma análise hidrológica de forma direta.

Chagas et al. (2010) detectaram que os MDEs ASTER GDEM e SRTM apresentam uma elevada quantidade de depressões espúrias. E se não há um preenchimento prévio destas falhas, pode-se gerar uma rede de drenagem descontínua (TARBOTON et al., 1991), o que inviabilizaria uma análise hidrológica proveniente desta fonte de informação. Hengl et al. (2004) reiteraram que em aplicações hidrológicas, a qualidade dos parâmetros do terreno pode ser melhorada por ajuste de interpolação, com auxílio de uma rede de drenagem existente, ou por remoção das depressões espúrias.

Nota-se que o assunto sobre consistência de modelos digitais de elevação para aplicações hidrológicas ainda é pouco explorado na literatura técnica. Zhang et al. (2013) propuseram um novo método para consistência hidrológica de MDEs e, compararam-no com outros dois já existentes. Eles concluíram que o método proposto foi condizente com a informação hidrológica conhecida, pois menos canais paralelos foram gerados, em relação aos outros dois métodos comparados. No entanto, eles notaram uma dificuldade na determinação da rede hídrica consistente para áreas planas.

Oliveira et al. (2010) construíram um MDEHC (modelo digital de terreno hidrologicamente consistente) utilizando-se da técnica de aprofundamento da calha do rio. Para determinar a direção de fluxo foi utilizado o algoritmo D8 somente nos canais, enquanto que no restante do MDE utilizou-se o algoritmo D infinity, que subsidiou uma melhor definição dos processos de erosão hídrica da bacia. Os autores resolveram utilizar o algoritmo D8 nos canais, porque outros algoritmos eliminam a relação unimodal entre a direção do fluxo e a rede de drenagem, onde a transferência de fluxo é realizada de pixel para pixel. Eles destacaram que estes procedimentos têm melhores resultados quando aplicados em bacias hidrográficas com maiores declividades, sendo que o oposto gera incoerências na rede.

A importância sobre mais pesquisas nesta área é explicita, devido a problemas ainda sem solução, envolvendo principalmente a extração da drenagem, com qualidade, em áreas planas.

Neste contexto, este trabalho teve como objetivo a comparação qualitativa e quantitativa de parâmetros geomorfométricos extraídos de redes hidrológicas geradas a partir de superfícies originais (sem correções de qualquer tipo) e superfícies hidrologicamente consistentes. 


\section{FUNDAMENTAÇÃO TEÓRICA}

Neste tópico serão apresentados alguns conceitos como: métodos de interpolação; modelos digitais de elevação (MDE); construção de um MDT; MDEs Hidrologicamente Consistentes (MDEHCs) e algoritmos implementados nos programas SIG.Estes assuntos são essenciais para o entendimento do texto como um todo.

\subsection{Métodos de Interpolação}

Os programas SIG disponibilizam ferramentas projetadas a partir de algoritmos específicos para interpolar amostras formando uma superfície contínua. A lógica utilizada por estes interpoladores envolve geralmente, à distância e correlação espacial entre as amostras. Os interpoladores mais usadossão: Distância Inversa Ponderada do termo em inglês InverseDistanceWeighted (IDW), Spline e para análises hidrológicas o topo to raster também conhecido como ANUDEM.

Em termos matemáticos o interpolador IDW utiliza duas equações (1 e 2) em sua formulação, considerando a distância entre a célula que terá o valor interpolado e as amostras com valor conhecido. Além de um peso que é atribuído, ou seja, quanto mais próximo o ponto a ser calculado estiver da amostra, maior é o seu peso ou influência (GOODCHILD et al., 2005).

$$
\begin{gathered}
z(x)=\frac{\sum w i . z i}{\sum w i} \\
w i=1 / d_{i}^{2}
\end{gathered}
$$

Em que:

$z(x)$ - Valor a ser interpolado.

$z i-$ Valor conhecido.

$w i-$ Peso.

$d_{i}$ - Distância entre as amostras e o valor a ser interpolado.

O interpolador Spline estima valores em locais não amostrados por meio de uma função matemática que utiliza as amostras para interpolar curvas que podem passar exatamente sobre os pontos ou próximo deles (CARVER et al., 2006). Este método pode ser utilizado desde que não haja alterações abruptas entre pontos próximos do fenômeno analisado.

\subsection{Modelos Digitais de Elevação (MDE)}

No Brasil existe um projeto do Instituto Nacional de Pesquisas Espacial (INPE) denominado TOPODATA, que fornece um Modelo Digital de Elevação (MDE) e suas derivações básicas (declividade, hipsometria, orientação, sombreamento) com resolução espacial de 30 metros. Os dados digitais de elevação são obtidos a partir da aplicação de técnicas de geoestatística sobre dados SRTM 
originais disponibilizados pela National Aeronautics and Space Administration (NASA). Os arquivos processados e seus derivados podem ser adquiridos gratuitamente na Internet acessando-se o endereço http://www.dsr.inpe.br/ topodata/acesso.php

Os dados ASTER GDEM, são produzidos mediante um consórcio formado pelo METI - Ministry of Economy, Trade and Industry, do Japão e a NASA National Aeronautics and Space Administration. A intenção deste consórcio é disponibilizar um modelo digital de elevação para acesso livre e global. O ASTER GDEM, disponível na Internet desde junho de 2009 foi gerado com o uso de estereoscopia em imagens coletadas pelo instrumento ASTER sensor VNIR. A cobertura do GDEM estende-se na faixa latitudinal entre $83^{\circ}$ norte e $83^{\circ}$ sul, o que fornece um mapeamento de $99 \%$ da superfície do planeta (ABRAMS et al., 2010).

O mapeamento topográfico baseado em interferometria de dados oriundos de Synthetic Aperture Radar (SAR) é concebido por meio da captação de dados pelas antenas em ângulos de visada diferentes, considerando o não deslocamento da cena durante a coleta de dados. A técnica de interferometria de passagem única se baseia nas medições feitas por radares que estão em uma mesma plataforma separados por alguns metros (JENSEN, 2009).

A missão espacial Space Shuttle Radar Topography Mission (SRTM) lançada em 11 de fevereiro de 2000 utilizando o ônibus espacial Endeavour, contemplava uma antena banda $\mathrm{C}$ e uma banda $\mathrm{X}$ posicionadas no final de uma haste de 60 metros de comprimento (JENSEN, 2009).

Usando a informação de distância entre as duas antenas e as diferenças nos sinais de ondas retroespalhadas do radar, foi possível calcular a elevação da superfície da Terra. Durante o período da missão foram mapeados aproximadamente $80 \%$ da superfície do planeta, numa faixa latitudinal entre $60^{\circ}$ norte e $54^{\circ}$ sul (FARR et al., 2007).

\subsection{Construção de um MDT}

A superfície terrestre é caracterizada por uma infinidade de pontos com coordenadas tridimensionais, contudo, seria inviável utilizar todos na construção de um MDT em ambiente computacional. Neste caso, é preciso obter uma amostra deste total.

Uma das fontes de dados de altitude mais comumente utilizadas são as curvas de isovalor das cartas topográficas, analógicas ou digitais, que no Brasil, são construídas e distribuídas, de forma gratuita ou comercial, pelo Exército Brasileiro e Instituto Brasileiro de Geografia e Estatística (IBGE). Em ambiente SIG, é possível extrair curvas de nível e pontos cotados das cartas topográficas por meio de vetorização. Esses dados servem como base para o processo de interpolação.

Os pontos cotados nas cartas topográficas são amostras tridimensionais do terreno levantadas em trabalhos de campo. Essas amostras podem ser regulares, ou seja, a posição espacial $(\mathrm{x}, \mathrm{y})$ destas, mantêm um padrão de espaçamento nas direções $\mathrm{x}$ e $\mathrm{y}$. As amostras semirregulares preservam a regularidade em uma 
direção preferencial x ou y, enquanto que nas amostras irregulares não há um padrão de regularidade na distribuição e, na maioria dos casos, são as que melhor representam um fenômeno (FELGUEIRAS e CÂMARA, 2001).

A resolução espacial do grid (Figura 2), ou dimensões da célula, estão relacionadas com a distribuição das amostras. Se estas são regulares, cada célula terá armazenado um valor de altitude associado a uma posição bidimensional (x,y), a distância entre as amostras definirá a resolução espacial do grid. No entanto, se as amostras são semirregulares ou irregulares faz-se necessário o uso de métodos de interpolação para que cada célula não relacionada a uma amostra tenha o seu valor de altitude estimado (HENGL e REUTER, 2009; LI et al., 2010).

Considera-se modelagem de um MDT a obtenção da representação contínua da superfície a partir da interpolação de um conjunto de amostras tridimensionais (FELGUEIRAS e CÂMARA, 2001). De modo geral, a construção de um MDT depende de um conjunto de amostras e da técnica adequada de interpolação.

\subsection{MDEHCs e Algoritmos Implementados nos Programas SIG}

Encontram-se na literatura científica, vários termos, como correção hidrológica, consistência hidrológica ou condicionamento hidrológico, para denominar o procedimento de adequação de um MDE para análises hidrológicas. O fato é que estes diferentes termos se referem à geração de um Modelo Digital de Elevação Hidrologicamente Consistente (MDEHC) que é capaz de reproduzir com a máxima fidelidade o caminho preferencial do escoamento superficial (CHAVES, 2002; OLIVEIRA et al., 2010; OLIVEIRA et al., 2012; ZHANG et al., 2011).

Para alcançar o resultado de uma rede de drenagem conectada, O'callaghan e Mark (1984) sugeriram que o MDE fosse suavizado antes de gerar a matriz de direção de fluxo para reduzir as falsas depressões em quantidade e dimensão. $\mathrm{O}$ problema é que este procedimento gerou uma perda de informação do MDE e não garantiu a coincidência espacial entre a drenagem numérica e a real (MARTZ e GARBRECHT, 1992, 2000). Logo, surgiram novas técnicas mais elaboradas a fim de solucionar esta questão.

O método "Stream Burning" foi idealizado para tentar corrigir a não coincidência entre as redes de drenagem, combinando topografia digital e feições hidrográficas. Um valor constante de profundidade especificado pelo usuário é subtraído dos pixels que são interseccionados pelo vetor de drenagem, o que gera um rebaixamento no canal. Entre as principais vantagens deste método estão a sua simplicidade, eficiência computacional e a alteração de poucos pixels no terreno digital (HELLWEGER, 1997; MIZGALLEWICZ e MAIDMENT, 2009; SAUNDERS, 2000).

Pesquisas realizadas no Brasil e em outros países (Chaves, 2002; Pires et al., 2005; Moreira, 2006; Fernandes e Menezes, 2009; Tan e Xu, 2014) consideram o algoritmo ANUDEM como sendo o mais adequado na geração de feições hidrológicas, com preservação da integridade da paisagem.

O Austrália National University DEM (ANUDEM), idealizado por 
Hutchinson (1989), é um método que integra um algoritmo de drenagem forçada que remove falsas depressões utilizando uma técnica iterativa de interpolação por diferenças finitas, que foi projetado para ter a eficiência de métodos de interpolação local, como o IDW, sem perder a continuidade da superfície.

O algoritmo calcula a cada iteração uma grade com resolução espacial mais fina a partir de uma grade de resolução espacial baixa, até alcançar a resolução definida pelo usuário. Os valores iniciais de baixa resolução são adquiridos por meio da média das amostras fornecidas. Os valores dos pixels são atribuídos de acordo com o valor da amostra mais próxima. O método iterativo de Gauss-Seidel é utilizado para calcular os valores dos pixels onde não há amostras, ou seja, entre curvas de nível (TAN e XU, 2014). Este algoritmo está implementado no programa ArcGis como topo toraster no módulo spatialanalyst.

\section{MATERIAL E MÉTODOS}

A área de estudo encontra-se na região centro sul do município de Goiânia, situado no estado de Goiás. A bacia hidrográfica do ribeirão Anicuns (figura 1), com área de aproximadamente $220 \mathrm{~km}^{2}$ possui mais que $60 \%$ de sua superfície, ocupada por áreas urbanizadas. De um total de 688 bairros encontrados na bacia, destacam-se alguns, como Setor Central, Campinas, Setor Leste Universitário, e Jardim Goiás, por serem bairros comerciais e de alta densidade habitacional.

Figura 1 - Localização da área de estudo.

MAPA DE LOCALIZAÇÃO DA BACIA DO RIBEIRÃO ANICUNS
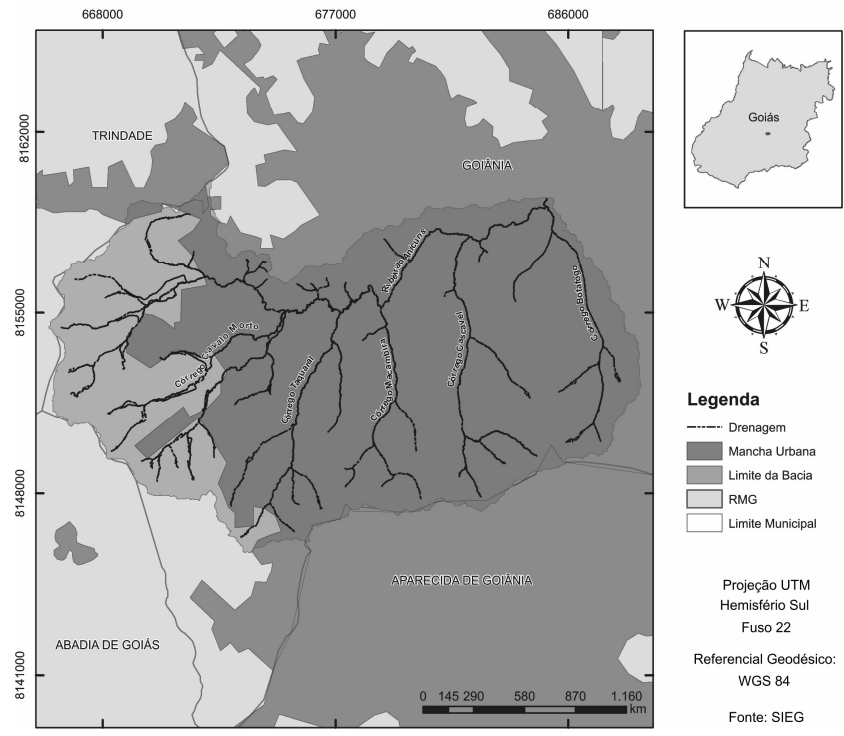

Bol. Ciênc. Geod. sec. Artigos, Curitiba, v. 20, no 4, p.784-805, out-dez, 2014. 
A bacia do Ribeirão Anicuns inserida na bacia do Rio Paraná, é constituída pelo Rio principal, o Ribeirão Anicuns, que é um afluente do Rio Meia Ponte, que por sua vez deságua no Rio Paranaíba, que é afluente do Rio Paraná. Os principais afluentes do Ribeirão Anicuns são: Córregos Macambira, Cascavel, Botafogo, Taquaral e Cavalo Morto, localizados na região central e mais a leste, de maior densidade urbana.

A maior parte da bacia tem um relevo que varia de plano à suave ondulado. Quatro tipos de solos estão presentes na região de estudo, Latossolos, Podzólicos, Cambissolos e Gleissolos segundo levantamento realizado em todo estado de Goiás por IPLAN/IBGE/UFG (1991).

A baixa declividade do relevo, em uma região densamente urbanizada do município de Goiânia, torna a bacia hidrográfica do Ribeirão Anicuns particularmente importante dentro do município de Goiânia, devido às grandes possibilidades de ocorrência de enchentes e alagamentos.

\subsection{Materiais Utilizados}

Foram utilizados cinco modelos de superfícies, dois modelos digitais de terreno (MDT) e três modelos digitais de elevação (MDE). Os MDTs de alta resolução foram produzidos por levantamento altimétricode varredura a laser e levantamento aerofotogramétrico, com resolução espacial de 1 metro e 5 metros respectivamente.

Dois MDEs considerados de média resolução espacial (30metros) o Topodata, elaborado pelo INPE, a partir da interpolação dos dados oriundos da missão SRTM (VALERIANO, 2005), e o Global Digital Elevation Model (GDEM), produzido por meio do consórcio entre o METI-Ministry of Economy, Trade and Industry, do Japão e a NASA - National Aeronautics and Space Administration.E por último um de baixa resolução espacial (90 metros) produzido pela missão Shuttle Radar Topography Mission (SRTM).

É importante destacar que o termo (MDT) modelo digital de terreno é reservado às superfícies produzidas com a altitude do solo, enquanto que, o termo (MDE) se refere às superfícies cuja altitude corresponde tanto à superfície do solo como dos objetos que estão acima dela, tais como o dossel das árvores e edificações. Deste ponto em diante, a fim de facilitar a leitura do artigo, quando houver a necessidade de citar todos MDTs e MDEs, será utilizado o termo superfícies.

Além destes dados, utilizou-se a hidrografia, oriunda do mapeamento urbano básico digital de Goiânia (MUBDG), como complemento do método de correção das superfícies, que será mais bem explicitado no decorrer do texto. O ArcGis9.3, Surfer 11 e Global Mapper foram os programas computacionais utilizados para o processamento dos dados.

\subsubsection{Modelo Digital de Terreno do Município de Goiânia}

O município de Goiânia possui dois modelos digitais de terrenos (MDT), o mais recente foi elaborado em 2011 por uma empresa privada de aerolevantamentos, 
que foi contratada pela prefeitura do município. Este MDT foi produzido a partir de levantamento aéreo, utilizando dispositivo de varredura a laser (LIDAR).

Para obtenção do MDT LIDAR utilizou-se a técnica de interpolação direta dos pontos altimétricos classificados como solo. Este MDT está armazenado em estrutura matricial com resolução espacial de 1 metro.

O outro MDT disponível no município foi elaborado em 2006, também por empresa especializada em aerofotogrametria, contratada pela prefeitura de Goiânia. Este MDT foi produzido a partir de observações altimétricas por meio de estereoscopia a partir de modelos aerofotogramétricos.

\subsection{Aplicações dos Métodos}

A priori determinaram-se dois grupos de informações. O original, derivado das superfícies sem nenhuma correção e o corrigido, derivado das superfícies hidrologicamente consistentes. As informações de drenagem foram extraídas de cada MDT e MDE, antes e após a correção, para uma posterior comparação entre as redes estimadas e a de referência.

Devido as diferentes resoluções espaciais envolvidas no estudo, utilizou-se como escala de comparação 1:100.000, que foi escolhida com base na menor resolução espacial do MDE SRTM. Isto torna possível comparar dados de maior resolução espacial com menor resolução espacial, tendo em vista que estes primeiros podem ser generalizados até certo ponto sem perdas significantes de informações. Caso contrário há perdas de informações.

A rede de drenagem MUBDG, utilizada no processo de rebaixamento da calha do rio, foi extraída a partir da Ortofoto de Goiânia na escala de 1:5000 por restituição aerofotogramétrica.

\subsubsection{Extração das Redes das Superfícies Originais}

As redes de drenagem para as superfícies originais, ou seja, sem alguma correção, foram extraídas segundo o conceito de O'Callaghan e Mark (1984), por meio do cálculo da direção de fluxo, fluxo acumulado e área de contribuição para cada célula dos MDTs/MDEs. Para tanto, utilizou-se o programa Surfer versão 11, que disponibiliza uma ferramenta para realização desta tarefa de forma rápida e precisa.

Ao final do processo, tem-se a rede de drenagem e limite da bacia hidrográfica, delimitados e armazenados em estrutura vetorial a partir de cada superfície original. De posse destes vetores foi possível caracterizar quantitativamente a rede de drenagem e área da bacia.

\subsubsection{Obtenção do MDEHC e Extração da Rede de Drenagem}

O procedimento de obtenção do MDEHC foi padrão para todas as superfícies, exceto para os MDTs. A alta resolução espacial destes dificultou a extração de atributos hidrológicos, uma vez que o algoritmo de direção de fluxo entende as vias urbanas como possíveis cursos d'água como ilustra a figura 2. 
Figura 2 - Rede de drenagem produzida a partir de um MDT de alta resolução sobreposta à camada de vias da região central de Goiânia.

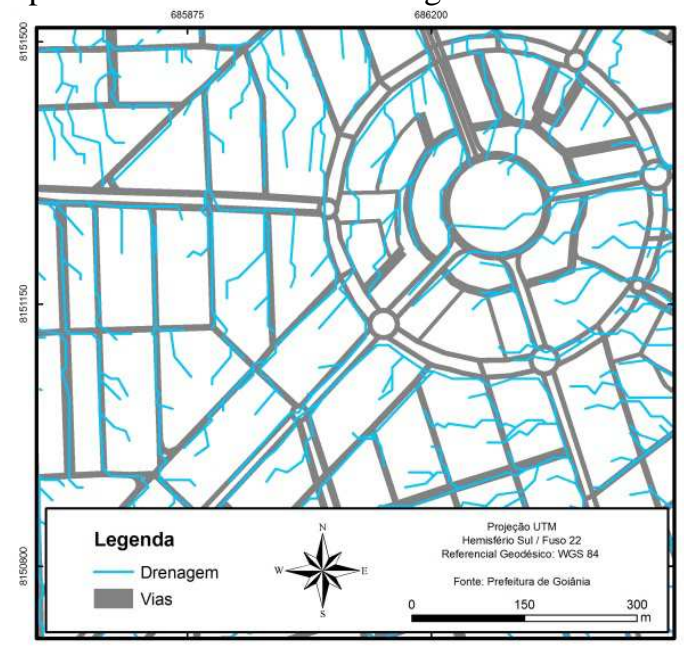

Buscou-se amenizar esta situação utilizando a interpolação e generalização do dado, alterando a sua resolução espacial de 1 metro para 5 metros. Como resposta obteve-se uma superfície mais suave. No primeiro momento o dado foi convertido da estrutura matricial para estrutura vetorial com feição pontual, onde cada ponto representa uma célula do MDT, com valor de altitude. Posteriormente utilizou-se o interpolador topo toraster para gerar o novo MDT.

O interpolador topo to raster é considerado uma primeira correção para as superfícies. Ele é um método de interpolação especificamente projetado para criação de modelos digitais de elevação hidrologicamente consistentes. Sua base vem do programa computacional ANUDEM (HUTCHINSON, 1988, 1989; ESRI, 2011). Em termos gerais o interpolador topo to raster elimina quase por completo as reentrâncias, possibilitando que o escoamento superficial chegue ao canal sem interrupções, além de desobstruir o caminho digital do canal "cortando" pontes ou artefatos que possam impossibilitar o livre escoamento da água.

Após esta correção inicial, procedeu-se com a técnica denominada stream burning ou o rebaixamento da calha do rio. É possível realizar esta ação de forma manual (Oliveira et al., 2007) ou de forma mais rápida, utilizando a extensão ArcHidro para ArcGis9.3, utilizando o comando DEM Reconditioning.

O objetivo desta ferramenta é ajustar a elevação da superfície para que a mesma seja consistente com a localização da rede de drenagem de referência, que neste estudo refere-se à rede de drenagem do MUBDG. Neste procedimento ascélulas coincidentes com a drenagem de referência são aprofundadas na superfície 
por um parâmetro definido pelo usuário. Deste modo, força-se o escoamento para dentro delas (HELLWEGER\& MAIDMENT, 1997).

Todavia, este processo ainda pode deixar algumas reentrâncias que precisam ser preenchidas antes da extração da rede de drenagem. Portanto, fez-se necessário utilizar a ferramenta fill do ArcGis 9.3, que elimina essas inconsistências. A partir deste ponto as superfícies podem ser consideradas hidrologicamente consistentes. $\mathrm{O}$ fluxograma ilustrado na figura 3 torna explícito o processo de obtenção do MDEHC e da rede de drenagem.

Figura 3 - Processo de obtenção da rede de drenagem por meio das superfícies hidrologicamente consistentes.

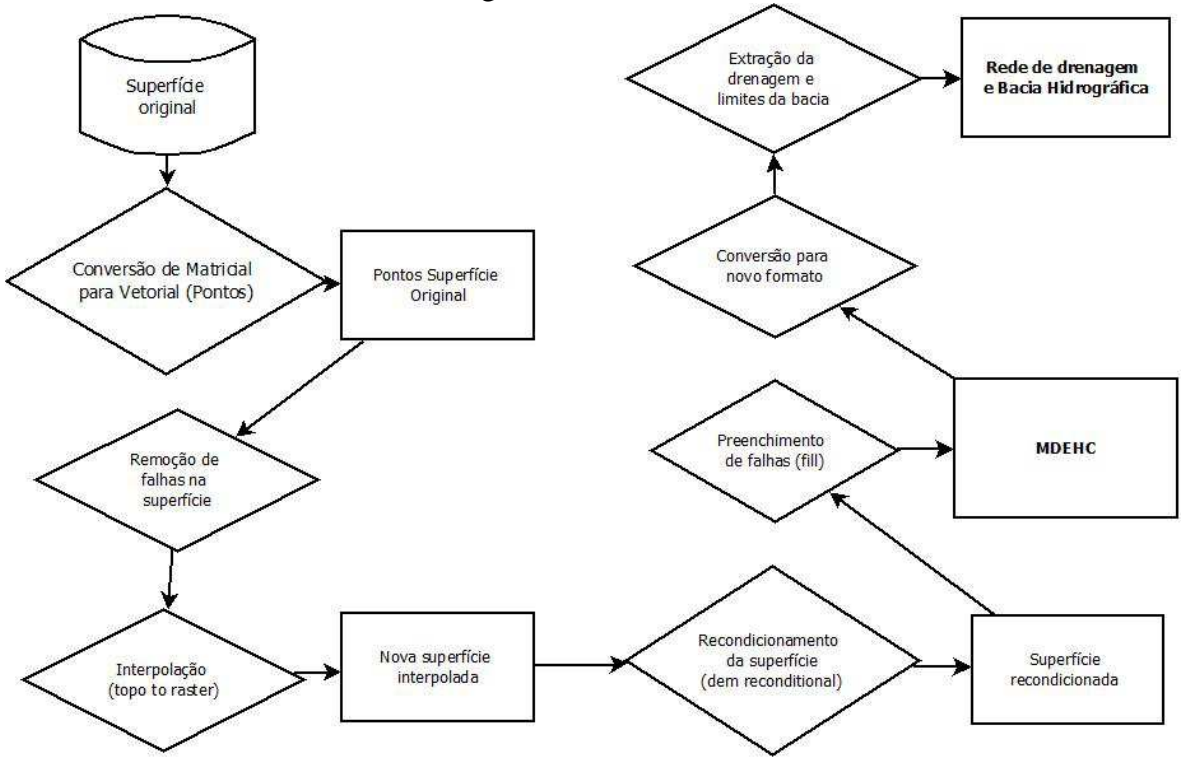

Ao executar as etapas do fluxograma ilustrado na figura 3 definiram-se as redes de drenagem e a bacia hidrográfica a partir de cada superfície hidrologicamente consistente. Em ambiente SIG foram organizados cinco arquivos com duas superfícies cada, uma com as drenagens a partir das superfícies originais e outra com as drenagens a partir das superfícies hidrologicamente consistentes. Isso facilitou a comparação e análise dos resultados. Com as informações organizadas prosseguiu-se com a extração dos parâmetros morfométricos.

\subsubsection{Caracterização da Rede de Drenagem e Avaliação de Discrepâncias}

$\mathrm{Na}$ etapa seguinte, buscou-se extrair alguns parâmetros morfométricos (tabela 1) para verificar o efeito do condicionamento das superfícies na extração da 
Ribeiro, H. J. ; Ferreira, N. C.

drenagem. Posteriormente, fez-se uma comparação qualitativa para avaliar o deslocamento da rede em relação à de referência.

Tabela 1 - Síntese dos parâmetros morfométricos para análise das alterações nas redes de drenagem.

\begin{tabular}{c|l|c|c}
\hline \multicolumn{2}{c}{ Parâmetros Morfométricos } & $\mathrm{km}^{2}$ & \multicolumn{1}{c}{ Referência } \\
\hline \multirow{4}{*}{ Forma da bacia } & Área & $\mathrm{km}$ & - \\
\cline { 2 - 4 } & Perímetro & - & - \\
\cline { 2 - 4 } & Fator de Forma & - & Villela e Mattos (1975) \\
\cline { 2 - 4 } & Índice de Circularidade & $\mathrm{km}$ & - \\
\hline \multirow{4}{*}{ Sistema de drenagem } & Comprimento do rio principal & $\mathrm{km}$ & - \\
\cline { 2 - 4 } & Comprimento total da hidrografia & $\mathrm{km} / \mathrm{Km}^{2}$ & Villela e Mattos (1975) \\
\cline { 2 - 4 } & Densidade Drenagem & $\mathrm{km}$ & - \\
\cline { 2 - 4 } & Comprimento do Talvegue & $\%$ & $\mathrm{Dt}=$ (hmax - hmin) / comprimento do talvegue \\
\hline \multirow{2}{*}{ Relevo da Bacia } & Declividade do Talvegue & & \\
\hline
\end{tabular}

Todos os parâmetros foram mensurados de forma direta. Para o cálculo dos índices seguiu-se a referência de Villela e Mattos (1975). No índice declividade do talvegue (hmax) e (hmin) representam respectivamente a altitude máxima e mínima na linha do talvegue.

Além destes parâmetros utilizou-se de outro artifício para verificar o quanto a rede extraída tanto pelas superfícies sem correção quanto pelas superfícies corrigidas se aproximou da rede de referência. Esta análise foi feita após a comparação visual.

Para executar esta análise, converteu-se cada rede da feição linha para feição pontual, em seguida utilizou-se a ferramenta de proximidade Near, que calcula a distância de cada pontoentre camadas de dados geográficos, que nesta pesquisa refere-se à drenagem de referência MUBDG e a drenagem estimada respectivamente. O cálculo das distâncias é adicionado na tabela de atributos da feição pontual, desta forma foi possível extrair os valores das distâncias médias para cada rede.

\section{RESULTADOS E DISCUSSÕES}

No gráfico da figura 4 encontram-se os resultados da caracterização morfométrica extraídos das redes de drenagem obtidas a partir das superfícies digitais. Os valores apresentados são resultantes da comparação entre a rede de drenagem obtida das superfícies originais, ou seja, sem nenhum tratamento ou correção e as superfícies hidrologicamente consistentes pelo método citado anteriormente.

Quantitativamente as maiores diferenças encontram-se no comprimento total da hidrografia, seguido do perímetro, declividade do talvegue e área. Este primeiro parâmetro tem influência direta no índice de densidade de drenagem, que, segundo (Vilella e Mattos, 1975), indica o grau de desenvolvimento de um sistema de drenagem, com valores que variam de $0,5 \mathrm{~km} / \mathrm{km}^{2}$ para bacias com sistema de 
drenagem pobre a $3,5 \mathrm{~km} / \mathrm{km}^{2}$ para bacias excepcionalmente bem drenadas. Para esta pesquisa o índice variou de 0,74 a $0,98 \mathrm{~km} / \mathrm{km}^{2}$, um valor considerado baixo, consequentemente pode-se dizer que a bacia possui um sistema de drenagem pobre.

Figura 4 - Caracterização morfométrica, realizada a partir das superfícies originais e corrigidas.

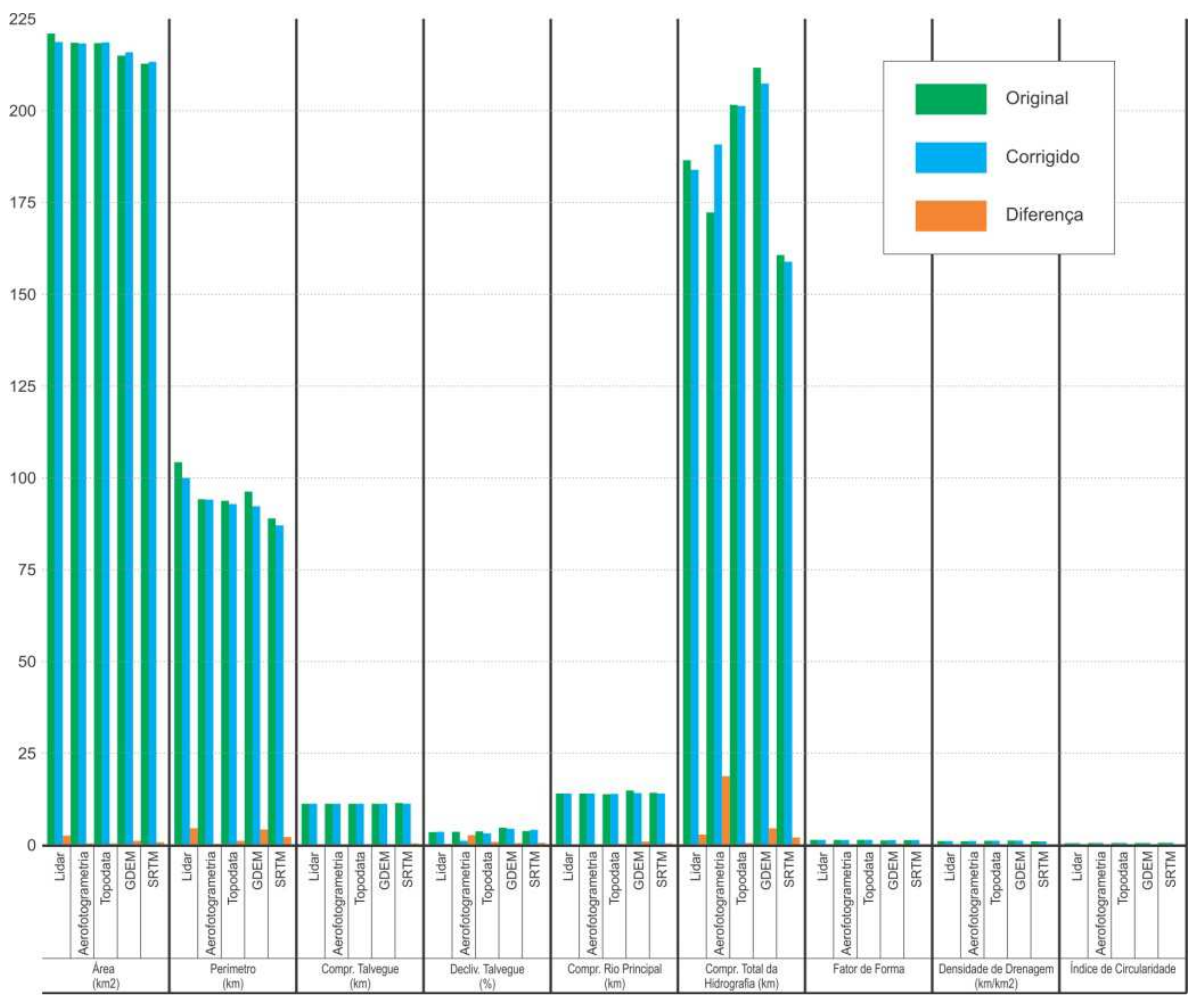

O relevo tem relação direta com o índice de densidade de drenagem. Linsleyet al. (1975) consideram que baixos valores para o índice refletem bacias com respostas hidrológicas lentas e relevo suave. Em relação às superfícies, a rede extraída a partir do MDT fotogrametria apresentou maior variação ao ser corrigida, em torno de $0,086 \mathrm{~km} / \mathrm{km}^{2}$ e o MDE Topodata a menor variação, com 0,002 $\mathrm{km} / \mathrm{km}^{2}$, o que sugere que a última superfície tem uma consistência hidrológica razoável.

Considerando os valores médios dos parâmetros analisados, os MDTs de alta resolução, aerofotogrametria e lidar, tiveram uma melhor resposta à correção aplicada. Quantitativamente os resultados não demonstram diferenças significativas, 
no entanto, vale salientar que na análise qualitativa as diferenças na forma da rede de drenagem são notáveis.

A figura 5 ilustra a comparação entre a rede de referência MUBDG e a rede numérica, extraída do MDT lidar sem correções e corrigido. O limiar usado na extração das redes geradas a partir dos MDTs de alta resolução (lidar e aerofotogrametria) foi de 25000 pixels.

Figura 5 - Sobreposição das redes de drenagem extraídas a partir do MDT Lidar com a rede MUBDG antes e após a correção. (A) rede drenagem extraída a partir do MDT Lidar original. (B) rede drenagem extraída a partir do MDT Lidar corrigido.

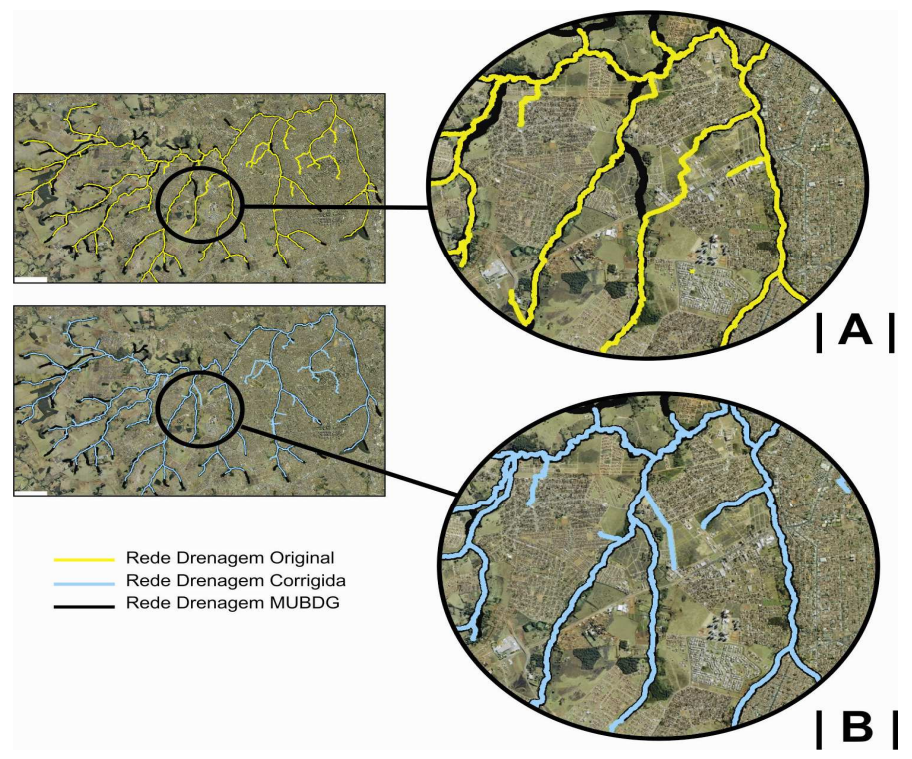

A rede de drenagem extraída do MDT sem correções teve um ajuste satisfatório à rede de referência, dada à alta complexidade do terreno, devido à densa urbanização local. No entanto, houve uma tendência na geração de canais em rodovias e vias em geral, como mostra o detalhe (A) da figura 5. A densa nuvem de pontos que varre a superfície consegue mapear objetos no solo, com precisão centimétrica, e mesmo com a aplicação de filtrosnão se consegue uma superfície suave o bastante para que não haja interrupção ou desvio do escoamento superficial em direção ao curso d' água. Além disso, em superfícies planas, há uma maior dificuldade no mapeamento de feições hidrológicas (CALLOW et al., 2007).

A correção aplicada ao MDT possibilitou uma coincidência espacial de aproximadamente $100 \%$ dos cursos d'água nos locais onde a rede foi coincidente. Nota-se na figura 5, observando-se os dois detalhes, a correção de um trecho da 
drenagem gerado em local não condizente com a realidade. Apesar da coincidência entre as redes, pode-se perceber a tendência na formação de alguns trechos de drenagem onde os mesmos não existem.

Na sequência da análise (figura 6) tem-se a comparação da rede de drenagem de referência com a rede de drenagem original e corrigida a partir do MDT elaborado por aerofotogrametria. É possível verificar que a rede derivada pelo MDT original, obtido por aerofotogrametria, apresentou uma maior inconsistência, comparado à rede original lidar.

Figura 6 - Sobreposição das redes de drenagem extraídas a partir do MDT elaborado pormapeamento aerofotogramétrico com a rede MUBDG, antes e após a correção. (A) rede drenagem extraída a partir do MDT Aerofotogrametria original.

(B) rede drenagem extraída a partir do MDT Aerofotogrametria corrigido.

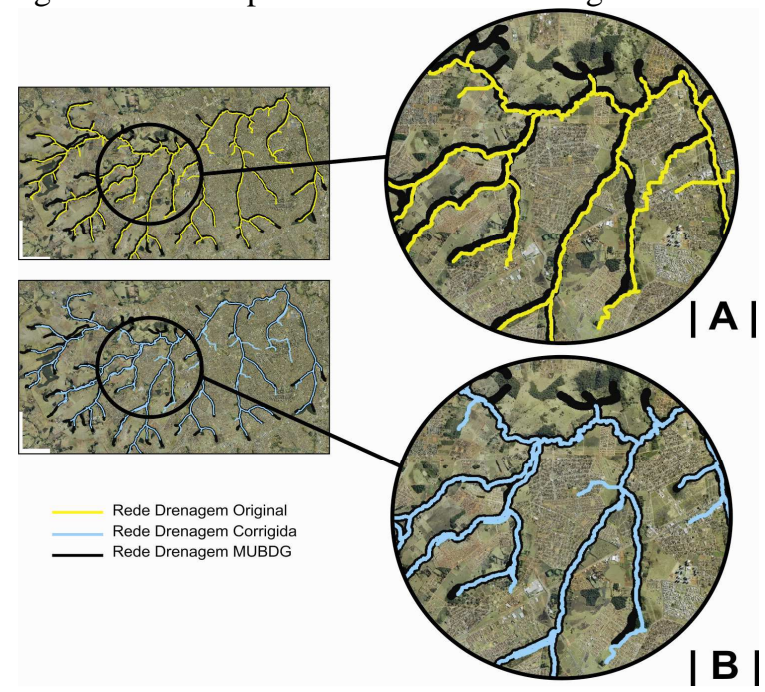

Foi possível observar que a rede de drenagem corrigida novamente apresentou uma coincidência espacial dequase $100 \%$, em relaçãoà rede de drenagem de referência. No detalhe B, nota-se na primeira confluência o deságue de dois canais existentes na hidrografia de referência e na corrigida, enquanto que, estes canais são generalizados para apenas um canal na hidrografia gerada pelo MDT sem correções (detalhe A). E ainda, pode-se visualizar o mesmo curso d'água, encontrado no MDT lidar, gerado sobre a rodovia, e também corrigido aqui.

O processo de aquisição do MDT por levantamento aerofotogramétrico é diferente do adquirido por mapeamento a laser. Entretanto, observa-se que existe uma dificuldade do algoritmo de direção de fluxo em delinear com precisão a rede hidrológica a partir de MDTs de alta resolução em ambientes urbanos. 
Dentre as superfícies pesquisadas, a de média resolução GDEM, teve o maior deslocamento entre a rede estimada e corrigida. Podem-se analisar estas divergências na figura 7 .

Figura 7 - Sobreposição das redes de drenagem extraídas a partir do MDE GDEM, com a rede MUBDG antes e após a correção. (A) rede drenagem extraída a partir do

MDE GDEM original. (B) rede drenagem extraída a partir do MDE GDEM corrigido.

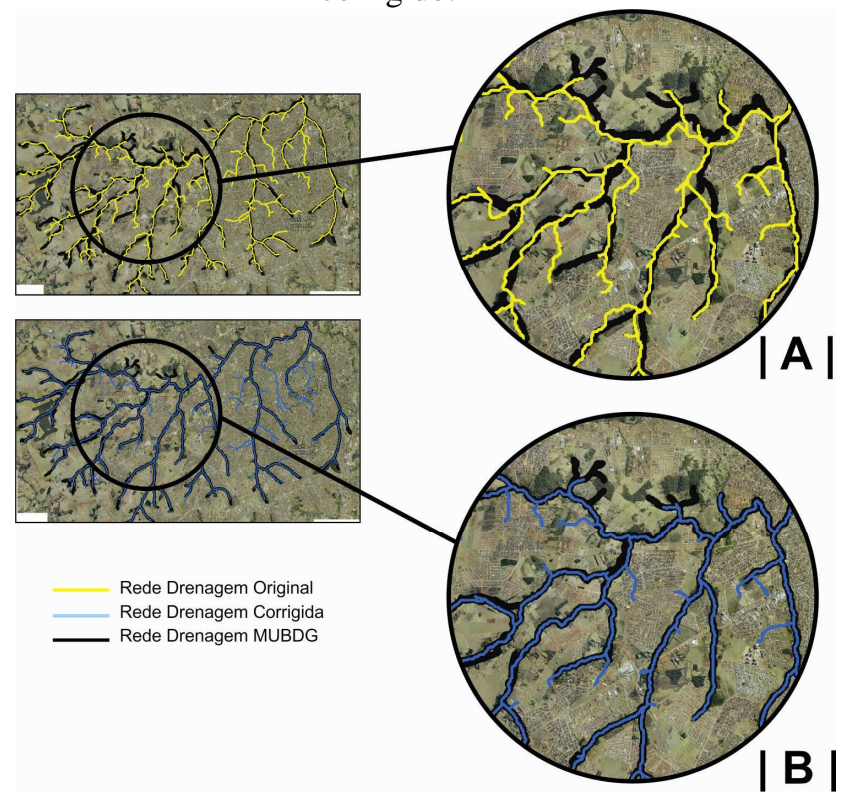

No detalhe (A) da figura 7, percebe-se um deslocamento visível entre a rede original e a de referência. Estas divergências são causadas, devido o método de aquisição da informação de solo. Tomazoni et al. (2011) também detectaram estas disparidades. Segundo eles o problema está na interferência causada pela vegetação, uma vez que o sistema imageador capta a resposta de qualquer objeto que esteja acima do solo e não necessariamente da superfície terrestre, onde se encontram os leitos dos corpos hídricos.

Porém é possível perceber que, ao aplicar a correção na superfície houve um ajuste satisfatório entre as redes. O condicionamento do MDE direcionou o escoamento para a correta localização dos cursos d'água.

A figura 8 apresenta a drenagem gerada a partir do MDE topodata. 
Figura 8 - Sobreposição das redes de drenagem extraídas a partir do DEM topodata à rede de referência MUBDG. (A) rede drenagem extraída a partir do MDE Topodata original. (B) rede drenagem extraída a partir do MDE Topodata corrigido.

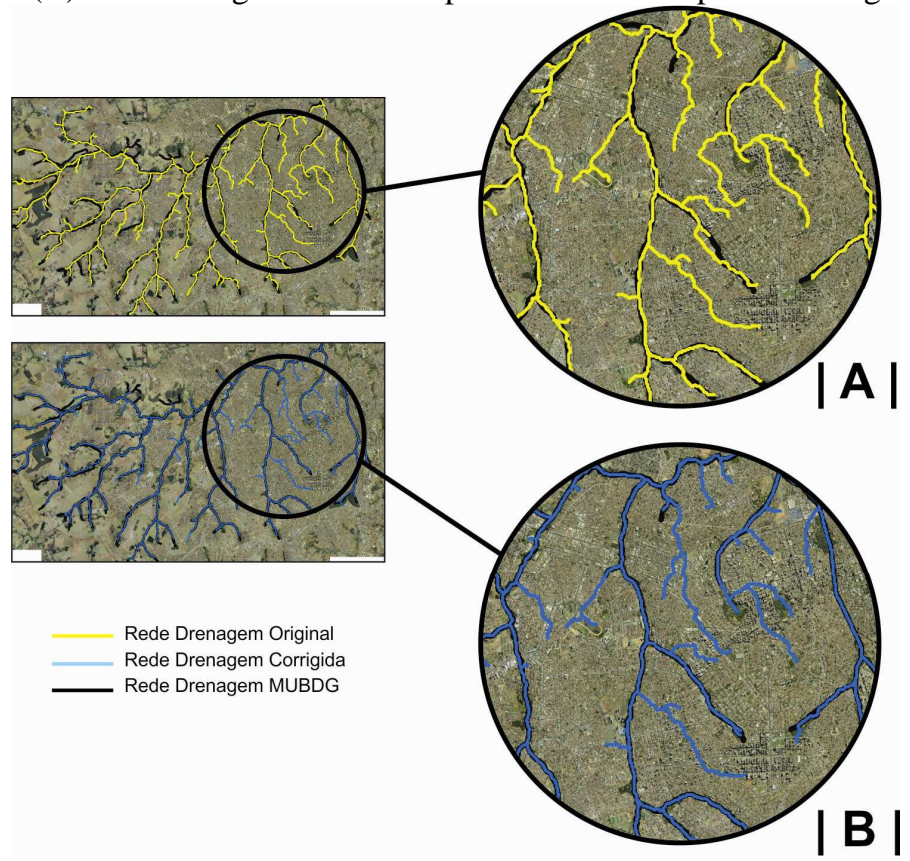

De modo geral a rede extraída do a partir do MDE topodata ajustou-se bem à rede de referência. Neste caso houve pouca divergência entre a rede estimada e a corrigida. Nas áreas com nível de urbanização mais intenso, houve uma tendência na formação de canais inexistentes. Explica-se a formação de falsos canais pelo mesmo princípio citado anteriormente no GDEM. Locais com uma grande densidade de objetos acima do solo podem gerar um MDE com valores de altitude que não representam a altitude do solo, e sim os alvos que estão sobre ele.

Para o MDE de baixa resolução SRTM reduziu-se o número de falsos canais em relação ao topodata e GDEM. Entretanto, o ajuste da rede gerada a partir do MDE original para a rede de referência não foi satisfatório, como ilustra a figura 9. A correção aplicada ao MDE SRTM direcionou o escoamento para a localização correta dos cursos d'água aumentando a consistência desta rede.

É válido destacar ainda na análise qualitativa que a coincidência espacial de aproximadamente $100 \%$ ocorre devido ao uso da rede MUBDG na etapa de rebaixamento da calha do rio utilizando a técnica Stream Burning. Ao extrair a rede numérica de uma superfície corrigida e compará-la com a mesma rede usada para 
correção, espera-se uma maior coincidência entre elas. Uma sugestão para novas pesquisas seria comparar as redes hidrográficas geradas a partir de superfícies hidrologicamente consistentes com outras redes de órgãos oficiais como IBGE por exemplo.

Figura 9 - Sobreposição das redes de drenagem extraídas a partir do MDE SRTM à rede de referência MUBDG. (A) rede drenagem extraída a partir do MDE SRTM original. (B) rede drenagem extraída a partir do MDE SRTM corrigido.

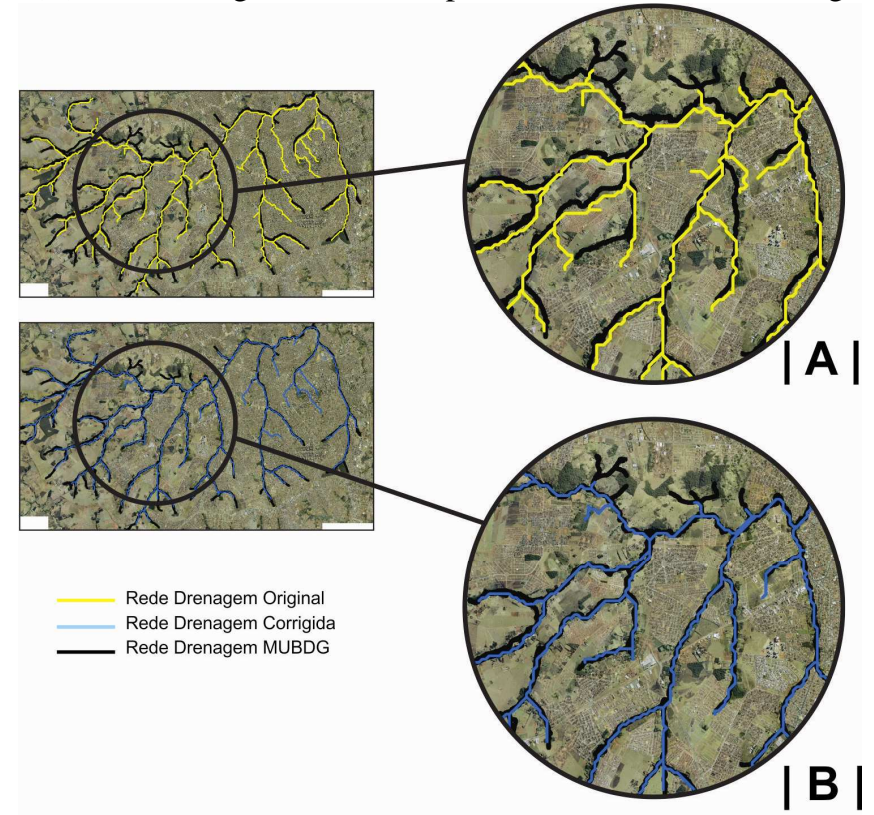

De forma a complementar a análise qualitativa elaborou-se o gráfico da figura 10, com as distâncias médias das redes geradas a partir das superfícies originais e corrigidas para a rede de referência MUBDG. A situação ideal para este tipo de análise seria o valor da distância média, igual ou próximo à zero. Neste caso hipotético, pode-se dizer que houve um ajuste de $100 \%$ entre as redes. Entretanto, consideraram-se as distâncias com toda a drenagem gerada e não somente a coincidente. Partindo desse pressuposto realizou-se a análise do gráfico.

A leitura do gráfico possibilita responder algumas questões da análise qualitativa. Percebe-se que as distâncias das redes são inversamente proporcionais a resolução espacial de cada superfície, ou seja, quanto menor a resolução espacial, maior o deslocamento, exceto para o GDEM, que mesmo com resolução espacial de 30 metros, não gerou melhores resultados do que o SRTM com resolução espacial de 90 metros. 
Figura 10 - Avaliação das distâncias médias entre as redes de drenagem geradas e a de referência.

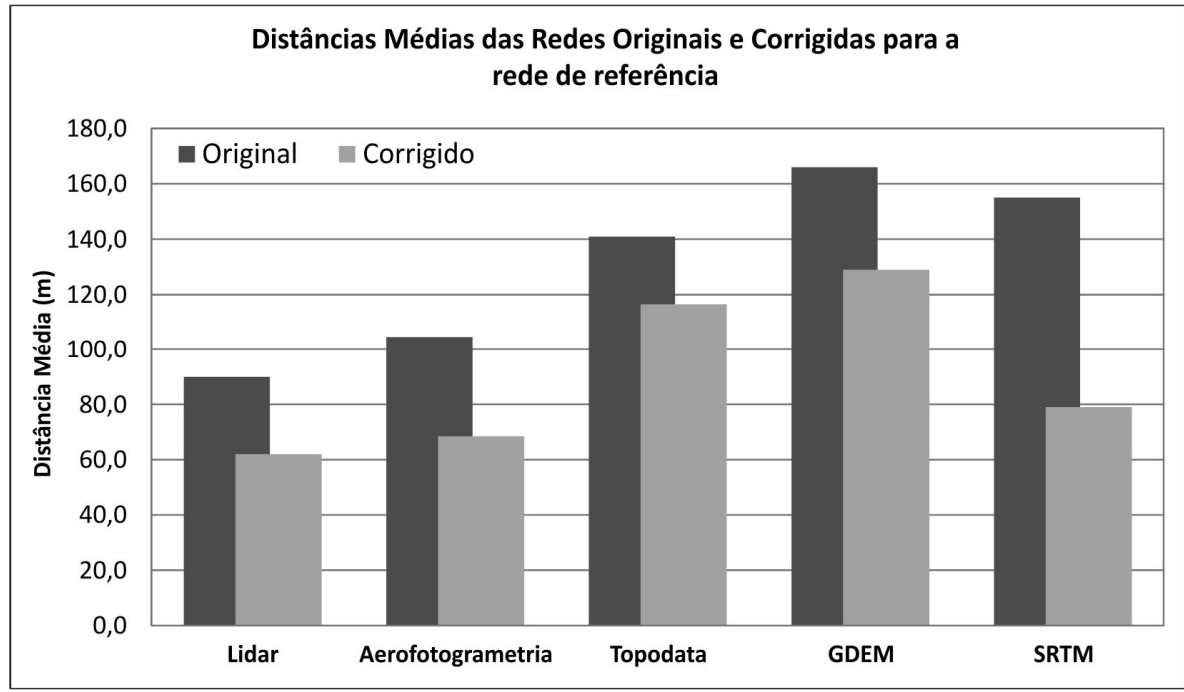

Das superfícies corrigidas, a Topodata foi a menos sensível a mudança, com uma amplitude da distância média (antes e após a correção) de apenas $24,5 \mathrm{~m}$. O SRTM teve a maior amplitude de ajuste da rede, com $76 \mathrm{~m}$. Os MDTs de alta resolução espacial (elaborados por mapeamento a laser e aerofotogrametria) tiveram resultados similares, com o maior ajuste para o MDT elaborado por aerofotogrametria. Vale ressaltar que o MDT lidar teve a sua resolução espacial generalizada de 1 metro para 5 metros, e o que se observa nas análises é um melhor desempenho desta superfície em relação ao MDT aerofotogrametria com resolução espacial compatível.

\section{CONCLUSÃO}

De modo geral, percebe-se que praticamente todas as redes hidrológicas estimadas a partir das superfícies sem correção tiveram tendências na geração de falsos canais, o que confirma a dificuldade atual de se definir feições hidrológicas em áreas relativamente planas. Essa dificuldade aumenta ainda mais, no caso de áreas de relevo plano, com intenso processo de urbanização.

Conseguiu-se um bom ajuste para os MDTs que produziram resultados similares, a escolha entre um destes para mapeamento hidrológico vai depender da escala de trabalho, que para este artigo foi acima de 1:100.000. Sugere-se que se façam outros testes observando o método aplicado em diferentes escalas.

Dentre os MDEs de média e baixa resolução o que se mostrou mais consistente foi o MDE Topodata, com menor amplitude de ajuste. No entanto, este gerou uma 
maior quantidade de falsos canais. Por outro lado, o MDE SRTM obteve o segundo melhor resultado, com uma coincidência na ramificação de canais mais compatível com a drenagem de referência.

Em aplicações hidrológicas, quando se dispõe de modelos digitais de terreno de alta resolução espacial, faz-se necessário a realização de generalizações e processamentos adicionais, considerando a rede hidrográfica, com o objetivo de minimizar os detalhes altimétricos decorrentes da urbanização, que podem prejudicar as análises hidrológicas.

\section{AGRADECIMENTOS:}

Os autores agradecem a CAPES e ao CNPq pelo apoio financeiro.

\section{REFERÊNCIAS BIBLIOGRÁFICAS}

ABRAMS, M.et al. The ASTER Global DEM. Photogrammetric Engineering and Remote Sensing, v. 76, n. 4, p. 344-348, 2010.

CALLOW, J. N.; et al. How does modifying a DEM to reflect known hydrology affect subsequent terrain analysis? Journal of Hydrology, v. 332, n. 1, p. 30-39, 2007.

CARVER, S. J.; et al.An Introduction to Geographical Information Systems.3 ed. Pearson Education Limited, 2006. 464 p.

CHAGAS, C. S. et al. Avaliação de modelos digitais de elevação para aplicação em um mapeamento digital de solos. Revista Brasileira de Engenharia Agrícola e Ambiental, v. 14, n. 2, p. 218-226, 2010.

CHAVES, M. A. Modelos digitais de elevação hidrologicamente consistentes para a bacia Amazônica. 2002. 111 f. 2002. Tese de Doutorado. Universidade Federal de Viçosa, Viçosa-MG, 2002.

ESRI, ArcGIS. 9.3 Help. Environmental Systems Research Institute (ESRI), http://webhelp. esri. com/arcgisdesktop/9, v. 3, 2011.

FARR, T. G. et al. The shuttle radar topography mission. Reviews of geophysics, v. 45, n. 2, 2007.

FELGUEIRAS, C. A.; CÂMARA, G. Modelagem numérica de terreno. FUKS, SD, CARVALHO, MS, CÂMARA, G. \& MONTEIRO, AMV Análise Espacial de Dados Geográficos. Disponível em:< http://www. dpi. inpe. br/gilberto/livro/analise/index. html>. Acesso em, v. 8, 2001.

FERNANDES, M. do C.; MENEZES, P. M. L. de. Comparação entre métodos para geração de MDE para a obtenção de observações em superfície real no maciço da Tijuca-RJ. Revista Brasileira de Cartografia, v. 2, n. 57, 2009.

GOODCHILD, M. F. et al. Geographic information systems and science. John Wiley and Sons, Chichester, 2005.539 p.

HELLWEGER, F.; MAIDMENT, D. AGREE-DEM surface reconditioning system.Maidment, DR (1997) (ed.)-GIS Hydro, v. 97, 1997. 
HENGL, T.; et al. Reduction of errors in digital terrain parameters used in soillandscape modelling. International Journal of Applied Earth Observation and Geoinformation, v. 5, n. 2, p. 97-112, 2004.

HENGL, T.; REUTER, H. I. (Ed.). Geomorphometry: concepts, software, applications. Elsevier, 2009.775 p.

HUTCHINSON, M. F. A new procedure for gridding elevation and stream line data with automatic removal of spurious pits. Journal of Hydrology, v. 106, n. 3, p. 211-232, 1989.

HUTCHINSON, M. F. Calculation of hydrologically sound digital elevation models. In: Proceedings of the Third International Symposium on Spatial Data Handling. Sydney, 1988.

IPLAN - Instituto de planejamento Municipal / IBGE - Instituto Brasileiro de Geografia e Estatística/ UFG - Universidade Federal de Goiás. Mapa de Solos. Carta de Risco de Goiás. Goiânia, 1991.

JENSEN, J. R. Sensoriamento remoto do ambiente. São José dos Campos. Parêntese Editora, 2009. $672 \mathrm{p}$.

LI, Z.;et al. Digital terrain modeling: principles and methodology. CRC press, 2010.266 p.

LINSLEY JR, Ray K.; et al. Hydrology for engineers.1 ed. McGraw-Hill, 1975.512 p.

MARTZ, L. W.; GARBRECHT, J. Numerical definition of drainage network and subcatchment areas from digital elevation models. Computers \& Geosciences, v. 18 , n. 6 , p. 747-761, 1992.

MARTZ, L. W; GARBRECHT, J. Digital elevation model issues in water resources modeling. Hydrologic and hydraulic modeling support with geographic information systems, p. 1-28, 2000.

MIZGALEWICZ, P.J; MAIDMENT, David R. Modeling agrichemical transport in midwest rivers using geographic information systems. 2009.

MOREIRA, M. C. Gestão de recursos hídricos: sistema integrado para otimização da outorga de uso da água. Viçosa, MG: UFV, 2006.

O'CALLAGHAN, J. F.; MARK, D. M. The extraction of drainage networks from digital elevation data. Computer vision, graphics, and image processing, v. 28, n. 3, p. 323-344, 1984.

OLIVEIRA, A. H. et al. Consistência hidrológica de modelos digitais de elevação (MDE) para definição da rede de drenagem na sub-bacia do horto florestal Terra Dura, Eldorado do Sul, RS. Revista Brasileira de Ciência do Solo, v. 36, n. 4, p. 1259-1267, 2012.

OLIVEIRA, S. N. de et al. Delimitação automática de bacias de drenagens e análise multivariada de atributos morfométricos usando modelo digital de elevação hidrologicamente corrigido. Revista Brasileira de Geomorfologia, v. 8, n. 1, 2010.

PIRES, J. M.et al. Análise da exatidão de diferentes métodos de interpolação para geração de modelos digitais de elevação e obtenção de características

Bol. Ciênc. Geod. sec. Artigos, Curitiba, v. 20, nº 4, p.784-805, out-dez, 2014. 
morfométricas em bacias hidrográficas. Revista Brasileira de Recursos Hídricos, v. 10, n. 2, p. 39-47, 2005.

SAUNDERS, W. Preparation of DEMs for use in environmental modeling analysis. Hydrologic and hydraulic modeling support with geographic information systems. ESRI Press, New York, p. 29-52, 2000.

TAN, Q.; XU, X. Comparative Analysis of Spatial Interpolation Methods: an Experimental Study. Sensors \& Transducers, v. 165, p. 155-163, 2014.

TARBOTON, D. G.; et al.On the extraction of channel networks from digital elevation data. Hydrological processes, v. 5, n. 1, p. 81-100, 1991.

TE CHOW, V.; et al. Applied hydrology.Tata McGraw-Hill Education, 1988. 540p.

TOMAZONI, J. C. et al. Uso de Modelo Digital de Elevação Gerados a partir do ASTER GDEM e SRTM para Caracterização da Rede de Drenagem do Município de Renascença no Sudoeste do Estado do Paraná (Use of Digital Elevation Model Generated from the SRTM and ASTER GDEM for...). Revista Brasileira de Geografia Física, v. 4, n. 2, p. 365-376, 2011.

VALERIANO, M. M. Modelo Digital de Variáveis Morfométricas com dados SRTM para o Território Nacional: O Projeto TOPODATA. Simpósio Brasileiro de Sensoriamento Remoto, v. 12, p. 3595-3602, 2005.

VILLELA, S. M.; MATTOS, A. Hidrologia aplicada. McGraw-Hill, 1975.245 p.

ZHANG, $\mathrm{H}$. et al. Uncertainty assessment of climate change impacts on the hydrology of small prairie wetlands. Journal of hydrology, v. 396, n. 1, p. 94103, 2011.

ZHANG, H.et al. Establishment of channel networks in a digital elevation model of the prairie region through hydrological correction and geomorphological assessment. Canadian Water Resources Journal, v. 38, n. 1, p. 12-23, 2013.

(Recebido em março de 2014. Aceito em agosto de 2014). 